\title{
Sur le théorème de v. Staudt et de Th. Clausen relatif aux nombres de Bernoulli.
}

\author{
(Par Niels Nielsen, à Copenhague.)
}

\section{Théorèmes généraux.}

Soit $p$ un nombre premier impair, tel que $p-1$ divise le positif entier pair $2 n$, nous disons pour abréger que $p$ soit du rang $n$.

Désignons ensuite par $m$ un positif entier quelconque, il est évident que le nombre premier $p$ du rang $n$ est du rang $m n$ aussi; c'est-à-dire que le nombre premier 3 est d'un rang quelconque.

Supposons, au contraire, donné le positif entier $n$, l'ensemble des nombres premiers du rang $n$

$$
\lambda_{1} \lambda_{2} \lambda_{3} \ldots \lambda_{v_{n}}
$$

est parfaitement déterminé.

Ces définitions adoptées, le théorème de v. Staudt $\left(^{*}\right)$ et de Th. GlauSEN $\left(^{* *}\right)$ donnera pour le $n$-ième nombre de Bernoulli une expression de la forme

$$
(-1)^{n} B_{n}=A_{n}+\frac{1}{2}+\frac{1}{\lambda_{1}}+\frac{1}{\lambda_{2}}+\cdots+\frac{1}{\lambda_{y_{n}}},
$$

où $A_{n}$ désigne un nombre entier.

Cela posé, il est érident que la détermination de la partie fractionnaire de $B_{n}$ exige la connaissance de tous les nombres premiers du rang $n$; c'est-à-dire qu'une telle détermination est un problème très difficile.

Quant à la partie entière $A_{n}$ du nombre $B_{n}$, elle dépend aussi des nom-

(*) Journal de Crelle, t. 21 , pp. 372-374; 1840.

(**) Astronomische Nachrichten, t. 17, col. 35̆1-352; 1840. 
bres premiers du rang $n$, comme le montrent clairement des théorèmes de v. Staudt $\left(^{*}\right)$ et de Stern $\left({ }^{* *}\right)$; mais la nature de ces nombres est parfaitement inconnue du reste.

On a indiqué un nombre de relations dites caractéristiques pour les $A_{n}$, relations qui sont illusoires considérées dans ce point de vue.

En effet, les formules en question ne sont autre chose que des propriétés communes aux coefficients d'un groupe très étendu des formules récursives pour les nombres de Bernoulut, ce qui est évident si nous étudions d'un point de vue général le problème susdit, sans nous borner à certains cas spéciaux.

A cet effet, nous prenons pour point de départ la formule récursive pour les nombres de Bernoull

$$
\sum_{s=1}^{\frac{n k-1}{q}}(-1)^{s} \alpha_{m, 2, x} B_{*}=\beta_{n}, \quad m \geqq 3 ;
$$

lésignons ensuite par

$$
p_{1} p_{2} p_{3} \ldots p_{\mu}
$$

l'ensemble des nombres premiers impairs égaux à $m$ au plus, puis introduisons dans (3) au lieu des $B$, les expressions tirées de (2), nous aurons une identité de la forme

$$
\sum_{s=1}^{\frac{m-1}{q}} \gamma_{m, 2 s} A_{x}=N_{m}-\sum_{q=1}^{q=\mu} \frac{M_{q u}}{p_{q}},
$$

où nous avons posé pour abréger

$$
\begin{aligned}
& N_{m}=\beta_{m}-\frac{1}{2} \sum_{s=1}^{\sum^{2}-1} \alpha_{m, 2 s}, \\
& M_{q}=\sum_{s=1}^{\leq p_{q}^{2}-1} \alpha_{m, s p_{q}-s} .
\end{aligned}
$$

(*) De unmeris Bernoullianis commentatio; De numeris Bernoullianis commentatio altera. Erlangue, 1845.

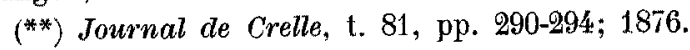


Cela posé, soit $2 p+1 \leqq m$ un positif entier impair, et soit ensuite

$$
\omega_{p}=\sum_{s=1}^{\frac{m-1}{q_{\rho}}} x_{m, q_{\rho}},
$$

il est évident que l'hypothèse $2 p+1=p_{q}$ entraîne l'égalité

$$
\omega_{p}=M_{q} .
$$

Considérons maintenant les équations

$$
\leqq \sum_{s=1}^{\frac{m-1}{2}} \alpha_{m, 2 s} A_{s}=N_{m}-\sum_{s=1}^{\frac{m-1}{2}} \frac{\omega_{s} \delta_{s}}{2 s+1} . \quad m \geqq 3,
$$

linéaires dans les nombres $\delta_{s}$, nous aurons en vertu de (5) le théorème suivant :

I. Posons dans (8) successivement

$$
m=3,5,7, \ldots, 2 p+1,
$$

il est possible de déterminer les $\rho$ nombres

$$
\delta_{1} \delta_{2} \delta_{3} \ldots \delta_{p}
$$

et nous aurons toujours $\delta_{p}=1$ ou $\delta_{p}=0$, selon que $2 p+1$ est un nombre premier ou non.

Revenons maintenant à la formule (5), puis supposons que $2 \beta_{m}$ et tous les coefficients $\alpha_{m, 2 s}$ soient des nombres entiers; il est évident que la somme qui figure au premier membre est un nombre entier. Remarquons ensuite que les dénominateurs qui figurent au second membre de la formule susdite sont sans diviseur commun, il faut que $M_{q}$ soit divisible par $p_{q}$; c'est-à-dire que nous aurons le théorème suivant:

II. Supposons entiers $2 \beta_{m}$ et tous les coefficients $\alpha_{m, 2 s}$, puis désignons par $p$ un nombre premier impair, égal à $m$ au plus, nous aurons

$$
\sum_{s=1}^{\frac{m-1}{p-1}} \alpha_{m, p s-s} \equiv 0 \quad(\bmod p) \text {. }
$$

Soit particulièrement $m$ un nombre premier, la formule (9) donnera pour $p=m$

$$
\alpha_{m, m-1} \equiv 0 \quad(\bmod m) .
$$


Le théorème inverse de II est évident, savoir :

III. Soient les coefficients $\alpha_{m, 9 .}$ des nombres entiers qui satisfont aux congruences (9), où p désigne un nombre premier impair, égal à $m$ au plus, l'expression

$$
2 . \sum_{s=1}^{\frac{m-1}{2}}(-1)^{s} \alpha_{m+2.2} B_{*}
$$

est toujours un nombre entier.

Or, il est très facile de généraliser beaucoup les deux derniers théorèmes.

A cet effet, désignons par $\gamma_{m, s_{s}}$ des nombres qui satisfont aux congruences

$$
\gamma_{m, 2 r} \equiv \gamma_{m, 2 s} \quad(\bmod p),
$$

où $p$ est uñ nombre płemier impair du rang $(2 r-2 s)$ et égal à $m$ au plus, nous aurons évidemment

$$
\sum_{s=1}^{\frac{m-1}{p-1}} \alpha_{m, n, s-s} \gamma_{m, p s-s} \equiv \gamma_{m, p-1} \cdot \sum_{s=1}^{\leqq \frac{m-1}{p-1}} \alpha_{m, p s-s} \quad(\bmod p) ;
$$

c'est-à-dire que la somme (11) peut être remplacée par cette autre

$$
\text { 2. } \sum_{s=1}^{\frac{m-1}{2}}(-1)^{s} \gamma_{m, 2 \times} \gamma_{m, 2 \times} B_{*} \text {. }
$$

Étudions encore la formule

$$
f(x)=K+\sum_{s=1}^{s=m-1} a_{m, s} s ! B_{s}(x),
$$

où les $B_{s}(x)$ désignent les fonctions de Bernoulu, de sorte que $f(x)$ est par conséquent un polynome entier du degré $m-1$, nous aurons l'autre théorème analogue à $\mathrm{II}$ :

IV. Supposons que tous les coefficients $a_{m, 2 s}$ de la formule (13) soient des nombres entiers, supposons ensuite que l'expression

$$
2 f(0)-2 K-a_{m, 1}
$$

ait la même propriété, nous aurons les congruences

$$
\leqq \sum_{s=1}^{\frac{m-1}{p-1}} a_{m, p s-s} \equiv 0 \quad(\bmod \nu)
$$

ò̀ $p$ désigne un nombre premier impair, égal à m au plus. 
En effet, posons dans (13) $x=0$, nous aurons la formule récursive

$$
K-\frac{1}{2} a_{m, 1}-f(0)=\sum_{s=1}^{\leq \frac{m-1}{v^{m}}}(-1)^{s} a_{m, 2 s} B_{*} ;
$$

il est digne de remarque que les coefficients $a_{m, 28+1}, s \geqq 1$, ne jouent aucun rôle pour les congruences (15).

\section{Applications diverses.}

Comme première application de nos théorèmes généraux, nous étudions la formule classique

$$
\sum_{s=1}^{\frac{m-1}{2}}(-1)^{s}\left(\begin{array}{c}
m \\
2 s
\end{array}\right) B_{s}=1-\frac{m}{2}, \quad m \geqq 3 ;
$$

le théorème II du paragraphe I donnera immédiatement les congruences

$$
\sum_{s=1}^{\frac{m-1}{p-1}}\left(\begin{array}{c}
m \\
p s-s
\end{array}\right) \equiv 0 \quad(\bmod p),
$$

où $p$ désigne un nombre premier impair, égal à $m$ au plus.

Supposons $m$ impair, savoir $m=2 n+1$, Ненмгте $\left(^{*}\right)$ a démontré directement les cas correspondants de la congruence (2), tandis que Lipschitz (**) a traité d'un autre point de vue la formule en question.

Désignons ensuite par

$$
p_{1} p_{2} p_{3} \ldots p_{\mu}
$$

l'ensemble des nombres premiers impairs, égaux à $m$ au plus, posons

$$
\Omega_{m}=\sum_{k=1}^{k=\mu} \frac{1}{p_{k}}\left(\sum_{s=1}^{\leqq \frac{m-1}{p_{k}-1}}\left(\begin{array}{c}
m \\
s p_{k}-s
\end{array}\right),\right.
$$

(*) Journal de Crelle, t. 81, pp. 93-95; 1876.

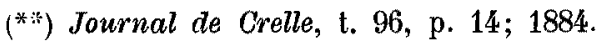


puis remarquons que la formule binomiale donnera

$$
\sum_{s=1}^{s=n}\left(\begin{array}{c}
2 n+1 \\
2 s
\end{array}\right)=2^{2 n}-1, \quad \sum_{s=1}^{n=n}\left(\begin{array}{c}
2 n+2 \\
2 s
\end{array}\right)=2^{2 n+1}-2
$$

nous aurons, en vertu de (1), ces deux formules

$$
\begin{aligned}
& \sum_{s=1}^{s=n}\left(\begin{array}{c}
2 n+1 \\
2 s
\end{array}\right) A_{s}=1-n-2^{2 n-1}-\Omega_{2 n+1}, \\
& \sum_{s=1}^{s=n}\left(\begin{array}{c}
2 n+2 \\
2 s
\end{array}\right) A_{s}=1-n-2^{2 n}-\Omega_{2 n+2},
\end{aligned}
$$

dont la première est due à Henmite.

Or, il est digne de remarque, ce me semble, que G.F. Meyer (*) déjà en 1862 a esssayé de développer la formule (4) d'Henмгте, mais qu'il n'a pas réussi à donner sous forme simple l'expression $\Omega_{2 n+1}$.

Soustrayons les deux formules (4) et (5), puis posons

$$
\Omega_{2 n+1}^{\prime}=\Omega_{2 n+2}-\Omega_{2 n+1}=\sum_{k=1}^{k=\mu} \frac{1}{p_{k}}\left(\sum_{s=1}^{\leqq} \sum_{k=1}^{\frac{2 n}{p_{k}-1}}\left(\begin{array}{c}
2 n+1 \\
p_{k} s-s-1
\end{array}\right)\right) .
$$

nous aurons la formule de $\operatorname{STERN}\left({ }^{*}\right)$

$$
\sum_{s=1}^{s=n}\left(\begin{array}{l}
2 n+1 \\
2 s-1
\end{array}\right) A_{s}=-2^{2 n-1}-\Omega_{2 n+1}^{\prime} .
$$

STEkn, dans son grand Mémoire intitulé: Beiträge zur Theorie der Eulerschen und Bernoullischien Zahlen (***), consacre les six dernières pages à une application analogue de ses trois formules récursives pour les nombres de Bermouler :

$$
\begin{aligned}
& \sum_{s=1}^{s=n-1}(-1)^{s}\left(\begin{array}{c}
2 n \\
2 s-2
\end{array}\right) B_{s}+(-1)^{n}\left(\left(\begin{array}{c}
2 n \\
2
\end{array}\right)-1\right) B_{n}=0, \\
& \sum_{s=1}^{s=n-1}(-1)^{x}\left(\begin{array}{c}
2 n \\
2 s-1
\end{array}\right) B_{s}+(-1)^{n}(2 n+1) B_{n}=-1 \\
& \sum_{s=1}^{s=n-1}(-1)^{*}\left(\begin{array}{l}
2 n-1 \\
2 s-2
\end{array}\right) B_{s}+(-1)^{n}(2 n+1) B_{n}=0
\end{aligned}
$$

(*) Archiv de Grunert, t. 38, pp. 241-246; 1862.

(**) Sournal de Crelle, t. 84, pp. 267-269; 1878.

$(\stackrel{* * *}{* *})$ Göttinger Abhandlungen, t. 23; 1878; 44 pages. 
formules qui satisfont évidemment aux conditions indiquées dans le théorème II du paragraphe I.

Désignons par $p$ un nombre premier impair, égal à $2 n+1$ au plus, nous aurons immédiatement les trois congruences

$$
\begin{aligned}
& \sum_{s=1}^{\sum_{p-1}^{2 n}}\left(\begin{array}{c}
2 n \\
p s-s-2
\end{array}\right) \equiv \delta_{p} \quad(\bmod p), \\
& \leqq \sum_{s=1}^{\frac{2 n}{p-1}}\left(\begin{array}{c}
2 n \\
p s-s-1
\end{array}\right) \equiv-\delta_{p} \quad(\bmod p), \\
& \leqq \sum_{s=1}^{\frac{2 n}{p-1}}\left(\begin{array}{c}
2 n-1 \\
p s-s-2
\end{array}\right) \equiv-\delta_{p} \quad(\bmod p),
\end{aligned}
$$

où il faut admettre $\delta_{p}=1$ ou $\delta_{p}=0$ selon que $p$ est du rang $n$ ou non; STERN démontre directement chacune des trois congruences en question.

Tels sont les résultats obtenus jusqu'ici en appliquant le théorème de v. Staudt et de Th. Clausen sur les formules récursives pour les nombres de Bernoulli.

Les trois auteurs susdits, savoir Hermite, Lipschitz et Stern, ont cru évidemment que ces résultats représentent des propriétés caractéristiques des coefficients binomiaux.

En effet, Hermite mentionne la fonetion numérique $\Omega_{m}$, définie dans la formule (3), du nombre $m$ dans le cas où $m$ est un nombre impair, savoir $m=2 n+1$. Or, il existe une fonction analogue du nombre pair $m=2 n+2$, et ces fonctions ne sont que des cas particuliers de la fonction générale

$$
\sum_{q=1}^{q=\mu} \frac{M_{q}}{p_{q}}
$$

qui figure au second membre de la formule (5) du paragraphe 1, pourvu que les conditions indiquées dans le théorème II du même paragraphe soient remplies.

LIPSGHITz donne le cas particulier de notre théorème général I du paragraphe I qui correspond à la formule (4) due à Hermite.

De plus, on dit que la même formule d'Hermite soit une formule récursive pour les nombres entiers $A_{n}$, quoique notre formule générale (5) du paragraphe I a précisément la même propriété. 
Or, considérons la Table des formules récursives pour les nombres de Bennoulur que j'ai donnée dans mon Mémoire $\left(^{*}\right)$ : Recherches sur les nombres de Bernoulli et d'Euler, nous verrons que la plupart de ces formules satisfont aux conditions susdites.

C'est-à-dire que l'application du théorème de v. Staudt et de Th. Cracsen sur de telles formules ne donne pas des propriétés caractéristiques des nombres entiers $A_{n}$, mais des propriétés communes des coefficients qui figurent dans les formules récursives susdites.

Nous nous bornerons à indiquer quelques autres applications de ce genre.

Posons pour abréger

$$
\lambda_{r}=\frac{1}{1}+\frac{1}{2}+\frac{1}{3}+\cdots+\frac{1}{r}
$$

nous aurons l'identité

$\frac{x^{m+1}+(-1)^{m}}{x+1}=(-1)^{m} \lambda_{m+1}+\sum_{s=0}^{s=m-1}(-1)^{s}\left[\left(\begin{array}{c}m+1 \\ s+1\end{array}\right)-1\right](m-s-1) ! B_{m-s}(x)$

puis différentions par rapport à $x$, l'hypothèse $x=0$ donnera la formule récursive

$$
\sum_{s=1}^{\frac{m-1}{2}}(-1)^{s-1}\left[\left(\begin{array}{c}
m+1 \\
2 s+1
\end{array}\right)-1\right] B_{s}=\frac{1}{2}+\frac{m(m-3)}{4},
$$

de sorte que nous aurons les congruences

$$
\sum_{s=1}^{\infty m-1}\left[\left(\begin{array}{c}
m+1 \\
p s-s+1
\end{array}\right)-1\right] \equiv 0 \quad(\bmod p)
$$

où $p$ désigne un nombre premier impair, égal à $m$ au plus.

Étudions encore les deux formules récursives (**)

$$
\begin{gathered}
T_{n+1}=\sum_{s=1}^{s=n}\left(\begin{array}{c}
2 n+1 \\
2 s
\end{array}\right) 2^{* *} B_{s} T_{n-s+1}, \\
n T_{w}=E_{n}-\sum_{s=1}^{\sum=n-1}\left(\begin{array}{l}
2 \\
2 \\
2^{2 n-2} s
\end{array}\right) 2^{2 *} B_{s} E_{n-s},
\end{gathered}
$$

(*) Mémoires de l'Académie Royale de Danemark, 1913. Dans ce qui suit je cite le Mémoire sous le titre donné dans le texte.

$\left.{ }^{(*)}\right)$ Recherches sur les nombres de Bernowlli et d'Euler, pp. 78-79; 1913. 
où les $T_{m}$ et les $E_{m}$ sont les coefficients des tangentes respectivement les nombres d'EULER, nous aurons pour le nombre premier $p=2 q+1$ :

$$
\begin{aligned}
& \sum_{s=1}^{\frac{n}{q}}(-1)^{q s}\left(\begin{array}{c}
2 n+1 \\
2 q s
\end{array}\right) T_{n-q s+1} \equiv 0 \quad(\bmod p), \\
& \leqq \sum_{s=1}^{n-1} \sum^{q}(-1)^{s}\left(\begin{array}{c}
2 n \\
2 q s
\end{array}\right) E_{n-q s} \equiv 0 \quad(\bmod p) \text {. }
\end{aligned}
$$

Remarquons encore que la somme qui figure au second membre de (14) est par conséquent un nombre pair, tandis que $E_{n}$ est toujours impair, nous aurons la proposition suivante:

I. L'expression

$$
\frac{n T_{n}}{2^{2 n-2}}=2\left(2^{2 n}-1\right) B_{n}
$$

est toujours un nombre entier impair.

Cette proposition due à Euler $\left(^{*}\right)$, est retrouvée par STERn $\left({ }^{* *}\right)$ el plus tard par WoRPIT\%KY $\left.{ }^{* * *}\right)$.

\section{Sur les coefficients de factorielles.}

Pour donner un exemple, dans lequel les conditions indiquées dans le théorème II du paragraphe I ne sont pas remplies, nous aurons à etudier les deux formules récursives $\left(^{* * * *}\right)$

$$
\begin{gathered}
\frac{n-r+1}{n+1} C_{n+1}^{r}-C_{n}^{r-\frac{n-r+1}{2} C_{n}^{r-s}}=\sum_{s=1}^{\leqq \frac{r}{n}}(-1)^{s-1}\left(\begin{array}{c}
n-r+2 s \\
2 s
\end{array}\right) C_{n}^{r-s e} B_{s}, \\
\frac{(n-1) !(n-1)}{2 n+2}=\sum_{s=1}^{n}(-1)^{s-1} C_{n}^{n-2 s} B_{s}
\end{gathered}
$$

(*) Institutiones calculi differentialis, p. 495; Saint-Pétersbourg, 1755. Opuscula Analytica, t. II, p. 273; Saint-Pétersbourg, 1785.

(**) Journal de Crelle, t. 88 , p. 92; 1880 .

${ }^{(* * *)}$ Journal de Crelle, t. 94, p. 232; 1883. Comparez la note de Kronecker dans le mêtme tome, p. 269.

${ }^{* * * *)}$ Recherches sur les nombres de Bernoulli et d'Euler, p. $66 ; 1913$. 
où les $C_{n}^{m}$ désignent les coefficients de factorielles, tandis qu'il faut admettre, dans (1), $2 \leqq r \leqq n-1$.

La formule (2) est due à Schömmah (*), mais retrouvée par A. Radicke (**). Étudions tout d'abord la formule (1).

Soit $p=2 q+1 \leqq r+1$ un nombre premier impair qui n'est pas diviseur de $n+1$, nous aurons la congruence

$$
\sum_{s=1}^{\frac{r}{2 q}}\left(\begin{array}{c}
n-r+2 q s \\
2 q s
\end{array}\right) C_{n}^{r-q s} \equiv 0(\bmod p), \quad 2 \leq r \leqq n-1
$$

Soit ensuite $n+1$ un nombre composé, et soit

$$
p_{1} p_{2} p_{3} \ldots p_{\text {!r }}
$$

l'ensemble des nombres: premiers qui divisent $n+1$ et qui ne dépassent pas $r+1$, nous verrons que le produit

$$
p_{1} p_{2} \ldots p_{\mu} \cdot \frac{n-r+1}{n+1} C_{n}^{r}, \quad 2 \leqq r \leqq n-1
$$

est toujours un nombre entier.

Soit entin $n+1=p$ un nombre premier, il est évident que $n+1$ et $n-r+1$ sont sans diviseur commun, et nous aurons par conséquent le théorème de Lagrange (***):

I. Soit $p$ un nombre premier impair quelconque, nous aurons

$$
C_{p}^{*} \equiv 0(\bmod p), \quad 1 \leqq r \leqq p-2 .
$$

Appliquons maintenant l'identité

$$
2 C_{p}^{z x+1}=\sum_{s=0}^{s=2 r}(-1)^{s}\left(\begin{array}{r}
p-s-1 \\
2 r-s+1
\end{array}\right) p^{2, \cdots-s+1} C_{n}^{s},
$$

cas particulier d'une formule générale que je viens de démontrer pour les polynomes réguliers $\left({ }^{* * * *}\right)$, nous aurons en vertu de $(5)$ cette autre proposition :

(*) Archiv de Grunert, t. 9, p. $234 ; 184 \%$.

(**) Die Recursionsformeln für die Berechnung der Bernoullischen und Eulerschen Zahlen, p. $15 ;$ Halle a. S., 1880.

(***) Mémoires de l'Académie de Berlin (1771), t. 2, pp. 125-137; 1773.

(****) Annali ali Matematica (3), t. 22, p. 78, formule (9); 1913. 
et de Th. Clausen relatif aux nombres de Bernoulli.

II. Soit $p>3$ un nombre premier impair, quelconque du reste, nous aurons

$$
C_{y}^{2 r+1} \equiv 0\left(\bmod p^{2}\right), \quad 1 \leqq r \leqq \frac{p-3}{2}
$$

Cela posé, introduisons dans (1) $2 r+1$ au lieu de $r$, puis remplaçons $n$ par le nombre premier $p$, nous aurons la congruence

$$
\frac{p-2 r}{p+1} C_{p+1}^{2 r+1}-\frac{p-2 r}{2} C_{p}^{2 .} \equiv(-1)^{r-1}\left(\begin{array}{c}
p-1 \\
2 r
\end{array}\right) C_{p}^{1} B . \quad\left(\bmod p^{2}\right) .
$$

Or nous aurons

$$
\begin{gathered}
C_{p+1}^{2 r+1}=C_{p}^{2 r+1}+p C_{p}^{2 r} \equiv 0 \quad\left(\bmod p^{2}\right), \\
C_{p}^{1}=\frac{p(p-1)}{2}, \quad\left(\begin{array}{c}
p-1 \\
2 r
\end{array}\right) \equiv 1 \quad(\bmod p),
\end{gathered}
$$

ce qui donnera finalement

$$
\frac{C_{p}^{\mathrm{g} r}}{p} \equiv \frac{(-1)^{r} B_{r}}{2 r} \quad(\bmod p), \quad 1 \leqq r \leqq \frac{p-3}{2} .
$$

Appliquons ensuite la formule récursive

$$
\begin{aligned}
& (-1)^{r}\left(C_{p}^{2 r+1}-\frac{(p-2 r-1) p}{2} C_{p}^{s r}\right)= \\
& \quad=\sum_{s=0}^{s=r-1}(-1)^{x}\left(\begin{array}{c}
p-2 s-2 \\
2 r-2 s
\end{array}\right) p^{2, \cdots 2 s} C_{p}^{2 s+1} B_{r-s},
\end{aligned}
$$

cas particulier d'une formule générale que je viens de démontrer pour les suites regulières $\left(^{*}\right)$, nous aurons

$$
\frac{C_{p}^{2 r+1}}{p^{2}} \equiv-\frac{(2 r+1) C_{z}^{2 r}}{p} \quad(\bmod p)
$$

c'est-à-dire que nous venons de démontrer le théorème suivant dû à M. Glaisher (**) :

III. Soit $p>3$ un nombre premier impair, quelconque du reste, nous

(*) Annali ali Matematica (3), t. 22, p. 86, formule (16); 1913.

$\left(^{* *}\right)$ Quarterty Journal of mathematics, t. 31, pp. 32L-353; 1900.

Annali di Matematica, Serie III, Tomo XXII. 
aurons les deux congruences modulo $p$ :

$$
\frac{C_{p}^{2 r}}{p} \equiv \frac{(-1)^{r} B_{r}}{2 r}, \quad \frac{C_{p}^{2 r+1}}{p^{2}} \equiv \frac{(-1)^{r-1}(2 r+1) B_{r}}{4 r},
$$

oì il faut supposer $1 \leqq r \leqq \frac{p-3}{2}$.

Étudions maintenant la formule (2).

Soit tout d'abord $n+1$ un nombre composé, le premier membre de la formule susdite est toujours un nombre entier; ce qui donnera

$$
\sum_{s=1}^{\frac{n}{p-1}} C_{n}^{n-p x+s}=0 \quad(\bmod p),
$$

où $p$ désigre un nombre premier impair égal à $n$ au plus.

Soit, au contratre, $n$ un nombre premier, nous remplaçons $n$ par $2 n+1$, ce qui donnera, en vertu de (7),

$$
(2 n) ! 2 n \equiv(-1)^{n-1} 2 n(2 n+1)(2 n+2) B_{n} \quad\left(\bmod (2 n+1)^{2}\right)
$$

Appliquons ensuite le résultat

$$
(-1)^{n-1}(2 n+1) B_{n} \equiv-1 \quad(\bmod 2 n+1),
$$

obtemu directement du théorème de v. Staud et de Th. Chausen, la formule (11) donnera immédiatement le théorème de Wrisson:

$$
(2 n) !+1 \equiv 0 \cdot(\bmod 2 n+1) .
$$

Posons pour abréger

$$
(2 n) !=(2 n+1) W_{n}-1,
$$

le nombre $W_{n}$ est un positif entier; nous le désignons comme quotient de WILSON.

Introduisons ensuite, dans (11), l'expression (13), il en résulte $2 n\left((2 n+1) W_{n}-1\right) \equiv(-1)^{n-1}\left(4 n^{2}+4 n\right)(2 n+1) B_{n} \quad\left(\bmod (2 n+1)^{2}\right)$, ou, ce qui est la même chose,

$$
2 n\left((2 n+1) W_{n}-1\right) \equiv(-1)^{n}(2 n+1) B_{n} \quad\left(\bmod (2 n+1)^{2}\right)
$$


de sorte que nous aurons

$$
2 n(2 n+1) W_{n} \equiv(2 n+1)\left((-1)^{n} B_{n}+\frac{2 n}{2 n+1}\right)\left(\bmod (2 n+1)^{2}\right)
$$

c'est-à-dire que nous avons démontré le théorème suivant:

IV. Soit $2 n+1$ un "nombre premier impair quelconque, le quotient correspondant de WILson satisfait à la congruence

$$
W_{n} \equiv(-1)^{n-1} B_{n}-\frac{2 n}{2 n+1} \quad(\bmod 2 n+1) .
$$

Ce théorème qui peut être considéré comme un supplément à celui de M. Glarsher est indiqué sans démonstration par M. Lerch (*).

Dans un Mémoire intitulé: Recheruhes sur les résidus quadratiques et sur les quotients de FERMa (**), j'ai donné d'autres démonstrations des deux derniers théorèmes et un grand nombre d'autres applications, sur la théorie des nombres, des nombres de Bernoultr, applications, qui sont nouvelles, je le crois.

Revenons encore une fois à la congruence (14) de M. Lerch, puis posons conformément au théorème de v. Staudt et de Th. Glausen

$$
B_{n}=\frac{a_{n}}{(4 n+2) B_{n}}
$$

où la fraction qui figure au second membre est irréductible, la formule (14) donnera après un calcul simple

$$
2 \beta_{n} W_{n} \equiv \frac{(-1)^{n-1} a_{n}+2 \beta_{n}}{2 n+1}-2 \beta_{n} \quad(\bmod 2 n+1)
$$

c'est-à-dire que nous aurons en outre la congruence

$$
(-1)^{n} a_{n} \equiv 2 \beta_{n} \quad(\bmod 2 n+1)
$$

où il faut supposer par conséquent que $2 n+1$ soit un nombre premier.

(*) Mathematische Annalen, t. 60, p. 488; 1905.

(*) Le Mémoire paraîtra dans les Annales de l'École Normale. 